\title{
Evaluation of Quality of Service in Smart-Hospital Communications
}

\author{
Angel Gomez-Sacristan ${ }^{1, *}$, Miguel A. Rodriguez-Hernandez ${ }^{2}$, and Victor Sempere ${ }^{3}$ \\ ${ }^{1}$ Dpto. Comunicaciones, Universitat Politècnica de València, Camino Vera s/n, 46022 Valencia, Spain \\ ${ }^{2}$ ITACA, Universitat Politècnica de València, Camino Vera s/n, 46022 Valencia, Spain \\ ${ }^{3}$ ITI, Universitat Politècnica de València, Camino Vera s/n, 46022 Valencia, Spain
}

\begin{abstract}
The concept of Smart-Hospital is generally associated with a comprehensive care model capable of responding to the needs of health institutions, companies and patients in an optimum way in terms of economic, operative and environmental aspects aiming the improvement of care quality and sustainable use of resources. In this context, a Smart-Hospital is a technological and hyper-connected hospital in terms of telecommunications. A huge range of systems and devices generate information of a heterogeneous nature. In many cases, for reasons of efficiency and availability, this information is stored and processed in architectures external to the hospital itself. Centralized services housed in Cloud architectures or telemedicine / tele-assistance services are proof of this. Guaranteeing an adequate level of quality of service is a complex task when approached from an analytical point of view due to the large number of sources and their heterogeneous nature. The use of simulation tools allows this task to be undertaken and using different hypotheses in less time and at a reasonable cost. This article presents the results obtained, in terms of quality of communications, for a Smart-Hospital with an arbitrary collection of heterogeneous services connected by Metro-Ethernet access. The results obtained: loss of information, delays and jitter will be used to outline the capacities to be contracted from the telecommunications supplier.
\end{abstract}

Keywords: Telemedicine, Smart-Healthcare, Telecommunications, Quality of Service, Simulation.

\section{INTRODUCTION}

In a Smart-Hospital, ${ }^{1}$ the ecosystem made up of people, equipment, servers, sensors and actuators is connected by cable, wirelessly or both, with different access points in the internal communications infrastructure. In this ecosystem a range of closely related processes coexist. These processes are difficult to define individually. The traditional services, including voice, multimedia and data, are evolving towards a convergent model $(\mathrm{NGN})^{2}$ in which the IP protocol has become a standard due to open-system, ubiquitous characteristics, its great flexibility and its low costs.

In this convergent, shared resource architecture, bandwidth consumption of a particular service may affect the working of another and moreover, critical information must have priority over less important information. This means that it is necessary to efficiently manage the information provided by users and devices, which have differing, random patterns of behaviour, traffic volume and temporal requirements, in order to ensure that each service satisfies the quality objectives as outlined in the international recommendations ${ }^{3,4}$ for this field. In convergent networks

*Author to whom correspondence should be addressed. different management and traffic prioritization mechanisms are used to carry out this task.

Analytically characterizing traffic generated from heterogeneous sources is a complex task, especially when their number is limited and their behaviour depends largely on the type of device in question and the service demanded. For example, in HTC (human type communications), temporal requirements are very strict, while in MTC (machine type communications) the number of sources can be very high. In many cases the problem can be resolved by contracting higher network capacity but this inevitably means higher costs. Thus, it is important to consider that simulation tools ${ }^{5}$ provide a flexible mechanism to solve this problem at a lower cost. In this article a simulation tool, designed specifically for networks with convergent access with heterogeneous traffic sources based on the IP protocol and applied to optimize the communications scenario of a Smart-Hospital, was used. This tool was designed using Omnet $++{ }^{6}$ simulation environment.

The paper is set out as follows: in Section 2, a general model of the services existing in a Smart-Hospital and the characteristics of the HTC and MTC traffic sources used is described; Section 3 shows the simulation scenario and the results obtained once the 


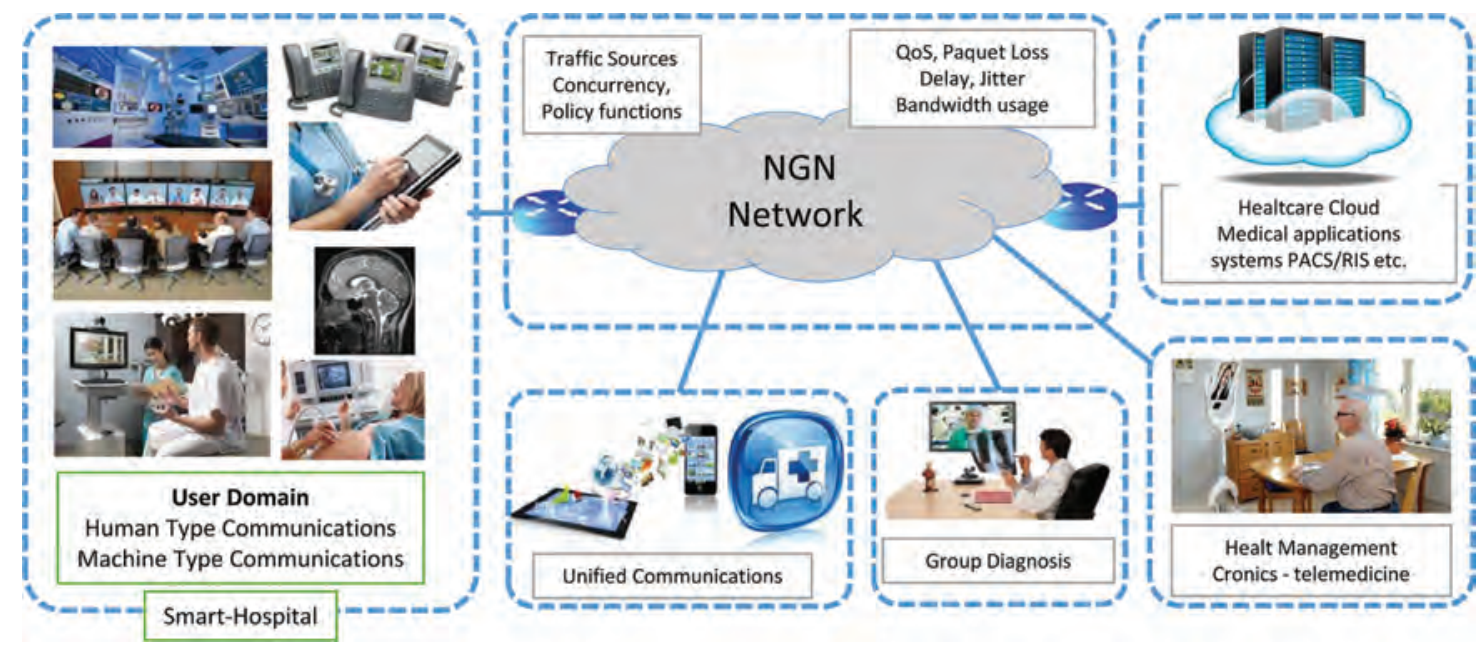

Fig. 1. Smart-hospital communications model.

Table I. HTC voice sources-multimedia QoS type.

\begin{tabular}{lccccc}
\hline Service & Sources & Codec & Data rate & Duration & Inactive time \\
\hline $\begin{array}{c}\text { Corporate } \\
\text { telephony }\end{array}$ & 200 & G.711 & $80 \mathrm{~Kb} / \mathrm{s}$ & $1-3$ minutes & $10-60$ minutes \\
$\begin{array}{c}\text { Call center } \\
\text { a }\end{array}$ & 20 & $\mathrm{G} .711$ & $80 \mathrm{~Kb} / \mathrm{s}$ & $1-6$ minutes & 2 minutes \\
\hline
\end{tabular}

resource needs were adequately outlined; finally the conclusions are presented.

\section{GENERAL SERVICES SCENARIO IN THE SMART-HOSPITAL}

Figure 1 shows the global connectivity environment for a SmartHospital in which a number of important systems exist, systems which have to be managed in a unified way in order to achieve efficient use of resources. Telemedicine based services ${ }^{7,8}$ collaboration between teams from different hospitals or the need for external management and storage systems that are accessible to all the health system create an extremely complex scenario.

Based on the external communication needs of a SmartHospital two categories of services have been defined: HTC (Human Type Communications) services in which there exists a need for real-time communication between people, such as voice and video-conference services; and MTC (Machine Type Communications) services, which are more related to information transfer, transfer of administration files, clinical histories, medical images, patient monitoring, etc. Within each category different subtypes can be defined. These subtypes have specific behaviour parameters to simulate different working environments. For example, the quality of the video conference during a surgery must be superior to that used in telemedicine services for chronic patients.

For each subtype, and based on how critical the service is, a category of quality can be designated (QoS). This means

Table II. HTC video sources-gold QoS type.

\begin{tabular}{|c|c|c|c|c|c|c|}
\hline Service & Sources & Quality & Motion & Data rate & Inactive time & Duration \\
\hline Videoconference & 5 & $\mathrm{HD}$ & Medium & $2.4-4.8 \mathrm{Mb} / \mathrm{s}$ & 20-40 minutes & 20-40 minutes \\
\hline Telepresence & 1 & $\mathrm{HD}+$ & High & $11-21 \mathrm{Mb} / \mathrm{s}$ & 20 minutes & $20-40$ minutes \\
\hline Telemedicine & 10 & $\mathrm{HQ}$ & Medium & $0.6-1.2 \mathrm{Mb} / \mathrm{s}$ & 10-20 minutes & 3-7 minutes \\
\hline
\end{tabular}

Table III. MTC data sources-gold and silver QoS type.

\begin{tabular}{|c|c|c|c|c|}
\hline Service & Sources & QoS type & Data sent by session (Bytes) & Sessions \\
\hline Medical sensors & 500 & Gold & $\begin{array}{l}0-10 \text { files of } 100 \mathrm{~B}-10 \mathrm{~KB} \\
0-2 \text { images of } 100 \mathrm{~KB}-500 \mathrm{~KB}\end{array}$ & 30 \\
\hline $\begin{array}{l}\text { Server upload } \\
\text { File transfers (HIS) }\end{array}$ & 100 & Silver & $\begin{array}{l}0-10 \text { files of } 10 \mathrm{~KB}-100 \mathrm{~KB} \\
0-2 \text { images of } 100 \mathrm{~KB}-500 \mathrm{~KB}\end{array}$ & 15 \\
\hline Cloud Services & 10 & Silver & $\begin{array}{l}0-10 \text { files of } 100 \mathrm{~KB}-10 \mathrm{MB} \\
0-5 \text { images of } 100 \mathrm{~KB}-500 \mathrm{~KB}\end{array}$ & 5 \\
\hline Dicom (RIS-PACS) & 5 & Silver & $\begin{array}{l}0-2 \text { files of } 100 \mathrm{~KB}-300 \mathrm{MB} \\
0-2 \text { images of } 1 \mathrm{MB}-30 \mathrm{MB}\end{array}$ & 5 \\
\hline Other medical equipment & 50 & Silver & $\begin{array}{l}0-5 \text { files of } 100 \mathrm{~KB}-10 \mathrm{MB} \\
0-2 \text { images of } 100 \mathrm{~KB}-1 \mathrm{MB}\end{array}$ & 10 \\
\hline Telemedicine & 100 & Silver & $\begin{array}{l}0-5 \text { files of } 10 \mathrm{~KB}-1 \mathrm{MB} \\
0-2 \text { images of } 100 \mathrm{~KB}-1 \mathrm{MB}\end{array}$ & 120 \\
\hline
\end{tabular}




\section{Table IV. QoS parameters.}

\begin{tabular}{lll}
\hline Traffic & \multicolumn{1}{c}{ CIR } & \multicolumn{1}{c}{ PIR } \\
\hline Multimedia & $2 \mathrm{Mbits} / \mathrm{s}$ & $4 \mathrm{Mbits} / \mathrm{s}$ \\
Gold & $40 \mathrm{Mbits} / \mathrm{s}$ & $80 \mathrm{Mbits} / \mathrm{s}$ \\
Silver & $20 \mathrm{Mbits} / \mathrm{s}$ & $100 \mathrm{Mbits} / \mathrm{s}$ \\
\hline
\end{tabular}

defining the degree of priority each type of traffic will have over other types in the network: Multimedia for critical real-time traffic, used principally for voice services; Gold for real-time traffic, used principally for video and priority data; and Silver for traffic of lower priority which can be discarded in periods of congestion.

To obtain a general idea of the behaviour of traffic sources in a real environment, this behaviour can be modelled based on predetermined parameters depending on their nature and activity time. These parameters can be fixed or random/variable values using statistical distributions.

\subsection{Human Type Communications (HTC) Voice and Real Time Video}

The parameters used to define the behaviour of the voice sources are the IP telephony codec used, call duration, inactivity time, quality of service required and the number of resources available for each type. ${ }^{9,10}$ For voice sources two types of traffic were defined; one associated with corporate telephony, and the other with a call centre system for patient attention and telemedicine services. Table I shows the characteristics of these traffic sources along with total number of sources. The duration of the sources was modelled with a limited negative exponential distribution. Voice traffic is usually associated with "Multimedia" quality of service.

The parameters used to define the behaviour of the video sources are: the codec of the video used; signal duration; inactive time; image quality (SD: $320 \times 180$ pixels, HQ: $640 \times 360$ pixels, HD: $1280 \times 720$ pixels and HD+ with: $1920 \times 1080$ pixels); number of frames per second; movement factor (low, medium, high); and the number of resources available for each type defined. To calculate the traffic rate H.264 (bitrate) of the video signal (1) the Kush Gauge model was used: ${ }^{11}$

$$
\text { bitrate }(\text { bits } / s)=v \times(\text { pixels } \times \text { frame_rate } \times \text { motion } \times 0.07)
$$

Where pixels represent the number of pixels per frame depending on the quality defined, frame_rate is the number of frames per second and motion is the movement factor ( 1 for low, 2 for

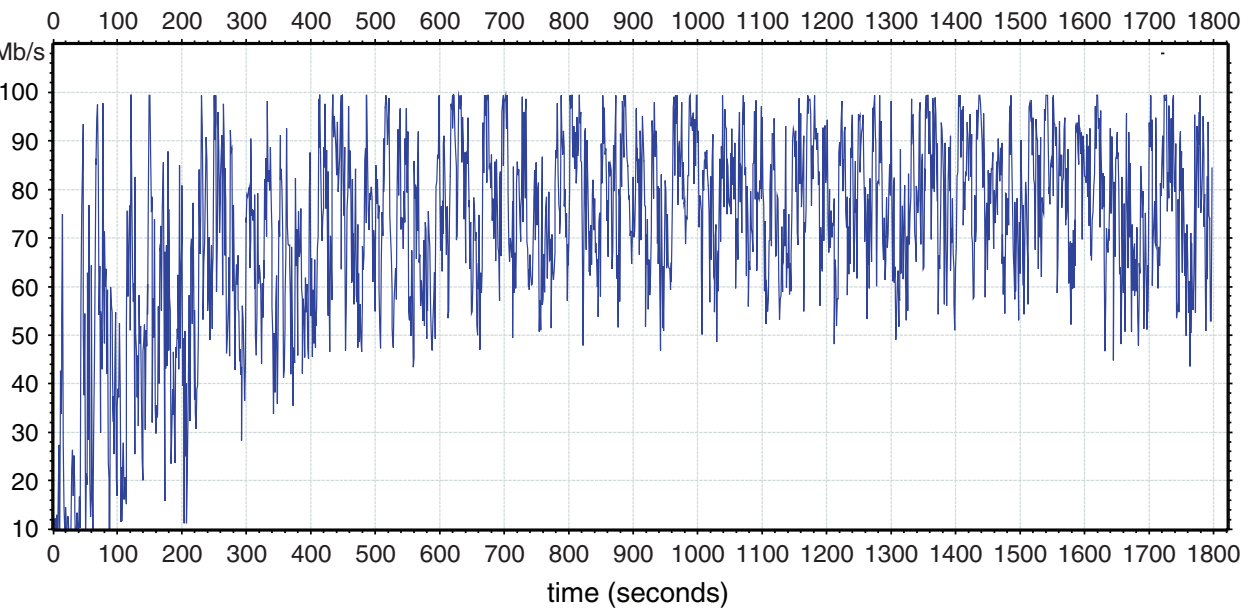

Fig. 2. Total hospital bandwidth usage.

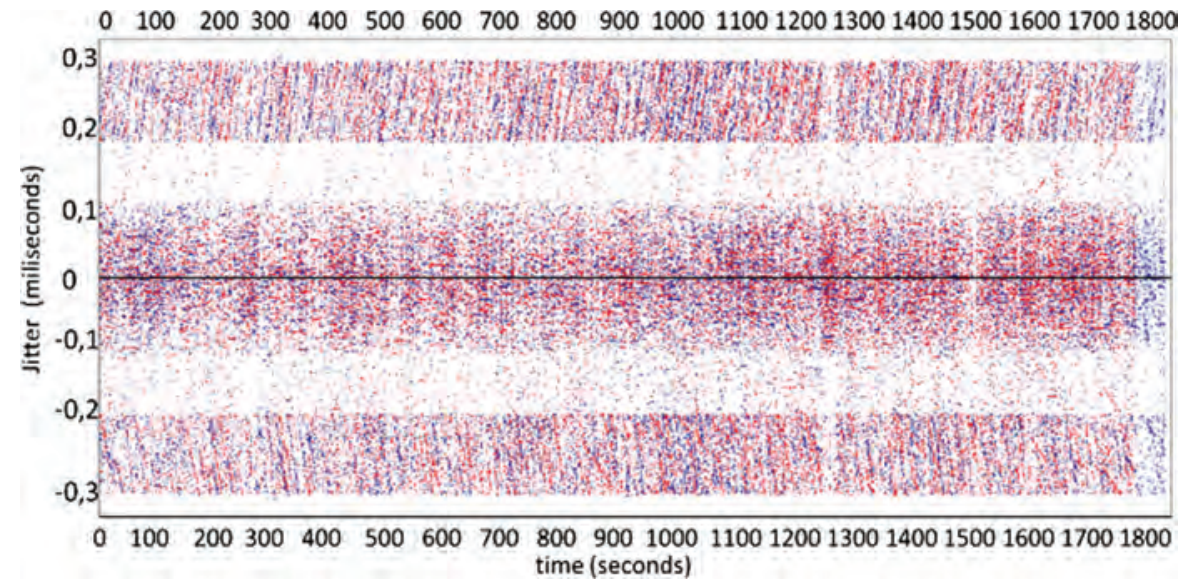

Fig. 3. Voice hospital jitter. 


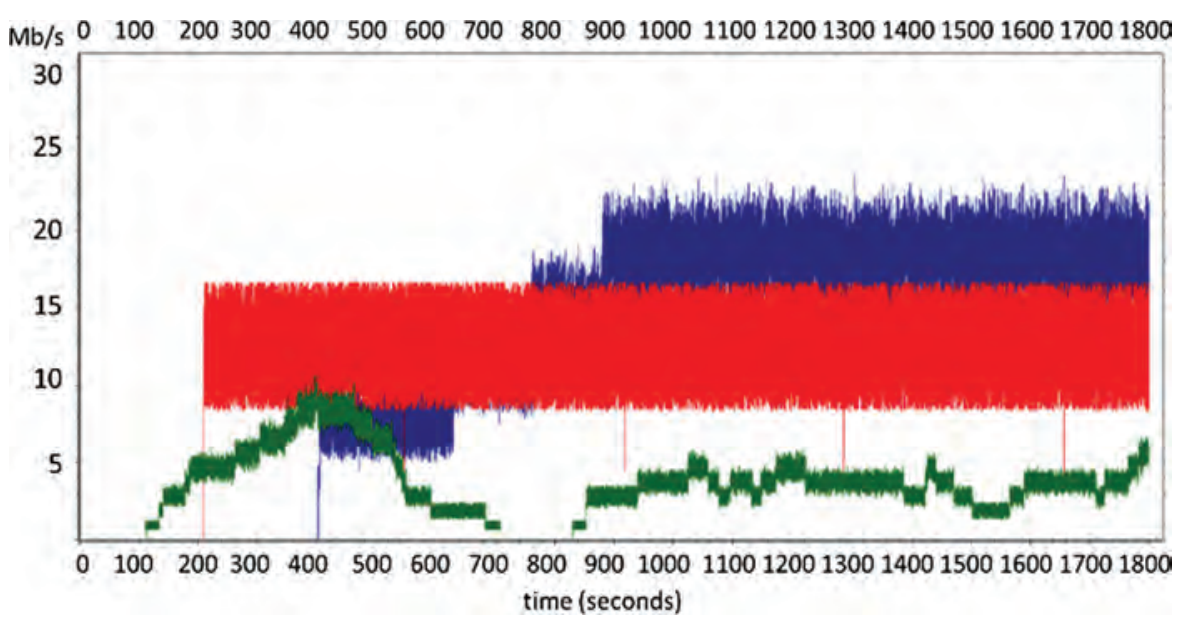

Fig. 4. Video hospital bandwidth usage.

middle and 4 for high). To simulate the random characteristics of the codec H.264 an uniform random variable " $v$ " has been added, which varies between 0.75 and 1.5 .

To simulate the video sources three types of traffic were defined and these can be seen in Table II: videoconference with HD quality; Telepresence (high-quality video conference) with HD+ quality; and finally video-telemedicine services with HQ quality. The duration of the sources was modelled with a limited negative exponential distribution.

Video traffic is usually associated with "Gold" quality of service.

\subsection{Machine Type Communications}

Traffic sources generated by diagnostic equipment, monitoring, access and file transfers, etc., are included in this category. The MTC sources can be characterized by activity time and the distribution of the data generated in this period. ${ }^{12}$ Sources of MTC traffic have been modelled as an arbitrary flow of information composed of " $s$ " windows of transmission or randomly distributed sessions in the simulation period. In each window, the source sends various blocks of information of variable size which simulate images and or data resources. In Table III, six different types of MTC sources simulating different services in the hospital are defined as an example. The number of files, images and their size were modelled using a limited negative exponential distribution.

However, each MTC source can be assigned in a flexible way to a quality of service category, according to the needs of the hospital. For instance, some RIS-PACS systems can be assigned to "Silver", but others used in operating theaters have to be assigned to "Gold".

\section{SIMULATION SCENARIO AND RESULTS}

Once the possible traffic generators have been described, the next step is to adequately outline the parameters of the WAN service offered by the operator. Taking as a reference the "Macrolan" service offered by the Spanish operator Telefonica ${ }^{13}$ and the Cisco catalyst-3650 switch-router, ${ }^{14}$ an interface with access of $100 \mathrm{Mb} / \mathrm{s}$ using Metro-Ethernet +MPLS technology was selected.

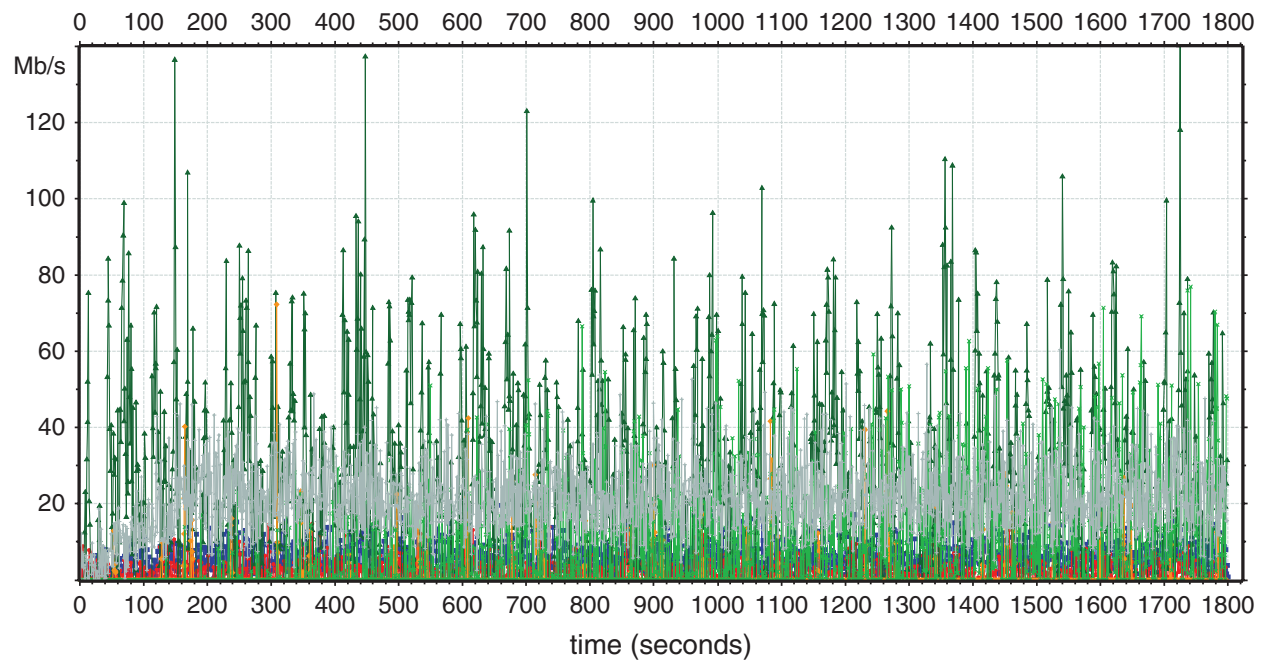

Fig. 5. MTC hospital bandwidth usage. 


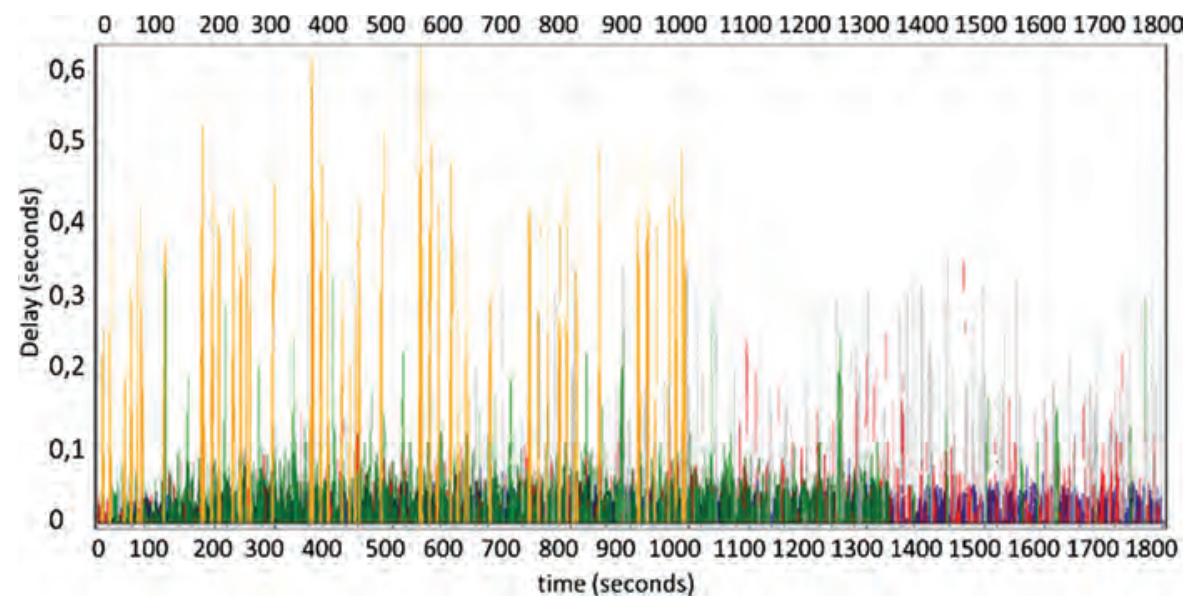

Fig. 6. Traffic delay for MTC sources.

As shown in the previous section, the service type "Multimedia" was assigned for voice traffic, "Gold" was assigned to video and critical sensors, and finally, "Silver was assigned to the remaining traffic. For each of the traffic types a collection of streaming parameters to be contracted from the operator were defined: CIR or guaranteed streaming; PIR or peak streaming; and others of less relevance such as queue management which are not mentioned here due to limitations on space. When the volume of traffic xceeds the CIR, but is less than the PIR, this is marked as "discardable" and is admitted to the network. If it exceeds the PIR, it can be discarded or assigned a lower priority where it will compete for available bandwidth with other streams of this type. In the "Silver" type service the traffic which exceeds the PIR is discarded.

The simulation tool allows the sources to be defined, the operator's access interface behaviour to be simulated and also allows regulation of simulation time and traffic measurement. ${ }^{15}$ Through a process of successive simulations of this scenario, an adjustment of streaming rate (CIR\&PIR) was carried out for each type of service with the aim of minimizing delays and jitter, as well as loss of information. Table IV shows the values obtained.

The simulation tool also allows detailed information on bandwidth consumption to be obtained for each type of service, as well as information on total bandwidth, delays and other parameters of interest.

A period of simulation of 1800 seconds was performed for the period of maximum activity with the concurrence of all traffic sources HTC and MTC with profiles defined in Table III.

Figures 2 to 7 show different graphs that reflect the behaviour of the network, bandwidth consumption for the HTC and MTC services, jitter for the voice sources, delays and information loss for the MTC sources.

Figure 2 depicts the use of bandwidth on the output interface to the network. As can be seen in this figure, during this period the average bandwidth consumed is close to $75 \mathrm{Mb} / \mathrm{s}$ with traffic peaks reaching the maximum interface capacity.

Figure 3 represents the delay variation (jitter) in voice HTC sources where it is possible to see how QoS mechanisms prioritizes this traffic over the others. In any case the maximum delay variations do not exceed 0.6 milliseconds.
Figure 4 depicts the bandwidth consumed by HTC video sources. The concurrence of five simultaneous HD video conferences generates traffic peaks of $23.6 \mathrm{Mb} / \mathrm{s}$. Telepresence service using HD+ codec generates a maximum of $16.4 \mathrm{Mb} / \mathrm{s}$. Lastly, video telemedicine services use a maximum of $10.6 \mathrm{Mb} / \mathrm{s}$. There is no loss of information because the statistical multiplexing produced by sources do not exceed the committed input rate of $40 \mathrm{Mb} / \mathrm{s}$ reserved for HTC video.

Figure 5 represents the bandwidth used by MTC sources. In order to illustrate the behaviour of the network during congestion periods, aggregate traffic sources were designed to exceed the $100 \mathrm{Mb} / \mathrm{s}$ that is the maximum bandwidth offered by the operator during certain periods. In these circumstances, the excess traffic assigned to the "Silver" class that cannot be carried out is stored in the lower priority output queue or discarded when this is full. The results of this congestion will produce increased delay and loss of information. Procedures using transport layer protocol (TCP/IP) allow retrieval of information discarded by QoS mechanisms, but this dramatically increases the delay.

Figure 6 shows the end-to-end delay of the MTC sources originating in hospital to the healthcare cloud. The maximum delay, including that introduced by the Telefónica MPLS network, and simulated according to the quality objectives set by the operator, does not exceed 0.7 seconds.

Finally, Figure 7 shows the volume of transmitted packets (in millions) and packet losses in percentage values. As can be seen

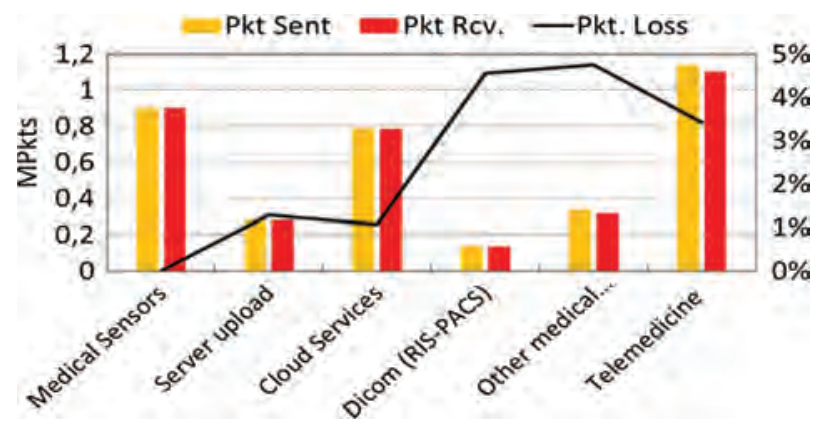

Fig. 7. Traffic loss for MTC sources. 
the figures, traffic sources with greater volume of information to be transmitted per session are the most affected although the total traffic volume is much lower than the rest.

\section{CONCLUSIONS}

Adequately designing the external communications resources for a Smart-Hospital leads directly to an improvement in patient care, and to a more efficient and sustainable use of resources. It is an analytically complex task due to the heterogeneous nature of the underlying services, and the ongoing evolution of related technology. However, simulation tools, such as the one described in this paper provide greater flexibility when defining the various scenarios with heterogeneous $\mathrm{HTC}+\mathrm{MTC}$ sources at a reasonable cost.

An arbitrary scenario of connectivity was designed with different types of traffic sources, its behaviour was simulated in a real operator environment and the minimum parameters that could be contracted were adjusted to guarantee an adequate level quality for HTC services. MTC services, those assigned to lower quality class, were dramatically affected with big end to end delays and packet losses.

As a future line of work, a greater number of HTC and MTC sources will be characterized, with the aim of increasing flexibility. Real HTC and MTC traces, extracted of real equipment, will also be included directly as specific traffic sources into the tool.

Acknowledgments: This work was supported by Spanish Government MEC Project TIN2013-47272-C2-1-R and by ITACA (Universitat Politècnica de València).

\section{References and Notes}

1. L. Yu, Y. Lu, and X. Zhu, Smart hospital based on internet of things. Journal of Networks 7, 1654 (2012).

2. International Telecommunication Union, ITU-T Recommendation Y.2001: Next generation Networks General Overview (2004).

3. International Telecommunication Union, ITU-T Recommendation Y.2112: A QoS control architecture for Ethernet-based IP access networks (2007).

4. International Telecommunication Union, ITU-T Recommendation Y.2113: Ethernet QoS control for next generation networks (2009).

5. K. Balasundaram, T. Velmurugan, and R. Suresh, Performance analysis of queuing disciplines for difference services using OPNET tool. International Journal of Scientific Engineering and Technology 3, 1 (2014).

6. OMNeT++ simulation framework http://www.omnetpp.org/.

7. T. Tanantong, E. Nantajeewarawat, and S. Thiemjarus, Toward continuous ambulatory monitoring using a wearable and wireless ECG-recording system: A study on the effects of signal quality on arrhythmia detection. Bio-Medical Materials and Engineering 24, 391 (2014).

8. J. Li, H. Y. Zhou, D. C. Zuo, K. M. Hou, and C. De Vaulx, Ubiquitous health monitoring and real-time cardiac arrhythmias detection: A case study. BioMedical Materials and Engineering 24, 1027 (2014).

9. R. Goleva, D. Atamian, S. Mirtchev, and D. Dimitrova, 3G network traffic sources measurement and analysis. Transactions on Emerging Telecommunications Technologies 25, 798 (2014).

10. A. D. Shaikh, K. J. Blow, M. A. Eberhard, and S. A. Fowler, Language independent on-off voice over IP source model with lognormal transitions, IET Communications 7, 1449 (2013).

11. H. Kush, 264 for the rest of us. available at: http://wwwimages.adobe.com (2009).

12. Service requirements for machine-type communications 3GPP TS 22.368 V1.2.2 (2010).

13. S. A. Telefonica, Macrolan service. Available at: http://www.movistar.es/ grandes-empresas/soluciones (2014).

14. Cisco Systems Inc., Cisco Catalyst 3650 Configuration Guide. available at: http://www.cisco.com/c/en/us/support/switches/catalyst-3650-seriesswitches/products-installation-and-configuration-guides-list.html) (2014)

15. T. Frantti and M. Majanen, An expert system for real-time traffic management in wireless local area networks. Expert Systems with Applications 41, 4996 (2014). 